Contato para correspondência: Hermínio Maurício da Rocha

Sobrinho

E-mail:

herminio.sobrinho@gmail.com

Conflito de interesse: Não

Financiamento: Recursos próprios

Recebido: 01/06/2021

Aprovado: 21/06/2021

\section{O USO DO MICROAGULHAMENTO ASSOCIADO AO DRUG DELIVERY NO REJUVENESCIMENTO CUTÂNEO: UMA REVISÃO DA LITERATURA}

\section{The use of microneedling associated drug delivery in cutane- ous rejuvenation: a literature review}

\author{
Raquel Costa', Luciana de Lara Pontes Ferreira', Patrícia Luz Almeida Leroy', \\ Hermínio Maurício da Rocha Sobrinho ${ }^{1,2}$ \\ ${ }^{1}$ Universidade Estadual de Goiás - UEG \\ 2 Pontifícia Universidade Católica de Goiás - PUC Goiás
}

\section{Resumo}

A associação do microagulhamento e o drug delivery tem se destacado com efeitos positivos por ser uma técnica que permite a administração transdérmica de fármacos, com menor tempo de inatividade e riscos relacionados ao paciente, pelo seu custo benefício e pelo excelente resultado. Este artigo trata-se de uma revisão bibliográfica narrativa que aborda os principais mecanismos de ação e efeitos biológicos do tratamento de microagulhamento associado ao drug delivery no rejuvenescimento cutâneo. $\mathrm{O}$ microagulhamento estimula a Indução Percutânea de Colágeno (IPC) ocasionando microlesões na derme que promovem uma inflamação controlada e a ativação de células produtoras de colágeno e elastina, proteínas estruturais que conferem firmeza e melhoram a aparência da pele. Estudos têm mostrado os benefícios do microagulhamento com drug delivery para administração transdérmica de medicamentos, facilitando o direcionamento de medicamentos até a derme e folículos pilosos. Conclui-se que o microagulhamento é um tratamento estético seguro, minimamente invasivo e eficaz para várias condições dermatológicas diferentes, incluindo acne, cicatrizes cutâneas, rítides, estrias e para o rejuvenescimento cutâneo. Dada a sua recuperação pós-tratamento conveniente, perfil de efeitos colaterais limitado e resultados clínicos significativos, o microagulhamento é uma valiosa alternativa a procedimentos estéticos mais invasivos.

Palavras-Chave: Microagulhamento; Rejuvenescimento; Drug delivery; Indução Percutânea de Colágeno.

\begin{abstract}
The association between microneedling and drug delivery has stood out with positive effects as it is a technique that allows the transdermal administration of drugs, with less downtime and risks related to the patient, due to its cost benefit and excellent result. This article is a narrative bibliographic review that addresses the main mechanisms of action and biological effects of microneedling treatment associated with drug delivery in skin rejuvenation. Microneedling stimulates Percutaneous Collagen Induction (IPC) causing microlesions in the dermis that promote controlled inflammation and the activation of collagen and elastin-producing cells, structural proteins that confer firmness and improve the appearance of the skin. Recent studies have shown the benefits of microneedling with drug delivery for transdermal administration of medications, facilitating the targeting of medications to the dermis and hair follicles. It is concluded that microneedling is a safe, minimally invasive and effective aesthetic treatment for several different dermatological conditions, including acne, skin scars, rhytides, striae for skin rejuvenation. Given its convenient post-treatment recovery, limited side effect profile and significant clinical results, the microneedling is a valuable alternative to more invasive aesthetic procedures. Keywords: Microneedling; Rejuvenation; Drug delivery; Percutaneous Collagen Induction.
\end{abstract}




\section{Introdução}

O rejuvenescimento cutâneo é um processo notável que envolve a restauração da integridade e da aparência da pele. Os procedimentos estéticos de rejuvenescimento vêm-se aperfeiçoando não apenas pelos avanços tecnológicos, mas também pela preocupação da população com a saúde, bem estar e a aparência física, bem como, em decorrência da maior longevidade ${ }^{1}$. No passado, a ideia de rejuvenescimento facial era dominada por procedimentos invasivos, especialmente técnicas de lifting facial².

A segurança e eficácia relatadas dos tratamentos com microagulhamento, inicialmente, tornou-se um procedimento atraente para pacientes que buscam um tratamento estético eficaz com o mínimo de tempo ocioso ${ }^{3}$.

O microagulhamento apresenta diversas indicações clínicas, especialmente, para o tratamento estético do rejuvenescimento cutâneo, de cicatrizes de acne e manchas, estrias cutâneas, facilitando a penetração de princípios ativos na pele 4 . Alguns estudos foram realizados para demonstrar que o microagulhamento afeta a administração de medicamentos, promovendo aumento da permeabilidade da pele, criando microcanais que estimulam o transporte transepidérmico/ transdérmico de formulações cosméticas ou fármacos ${ }^{5,6}$.

No tratamento com o microagulhamento a pele é cuidadosamente lesada na superfície e/ou em várias profundidades, através de um dispositivo com agulhas, induzindo a neocolagênese na derme, resultando no rejuvenescimento cutâneo ${ }^{4}$. A técnica de administração de medicamentos (drug delivery) otimiza os resultados do microagulhamento, chamando a atenção para o benefício da associação de procedimentos estéticos, levando resultados promissores ocasionados pelo aumento da permeabilidade do estrato córneo da pele ${ }^{5,6}$.

O objetivo deste estudo foi descrever os principais mecanismos de ação, efeitos biológicos e os benefícios da técnica do microagulhamento associado ao drug delivery no processo de rejuvenescimento cutâneo.

\section{Métodos}

Esta pesquisa é caracterizada como revisão bibliográfica narrativa. Para a busca dos estudos foram utilizadas as bases de dados virtuais: Portal de Periódicos Capes e US National Library of Medicine (PubMed) e livros científicos. Foram utilizados os seguintes Descritores em Ciências da Saúde (DeCS) ou palavras-chave, isolados ou combinados: microneedling, skin, roller, rejuvenation, drug delivery, nos idiomas português e inglês, publicados no período de 2005 a 2020. Foram incluídos artigos com textos completos disponíveis nas bases de dados utilizadas e que apresentaram conteúdos compatíveis com os objetivos propostos. Foram excluídos artigos duplicados, aqueles publicados fora do período temporal estabelecido, os não disponíveis integralmente nas plataformas virtuais e aqueles não condizentes com os objetivos deste estudo. $O$ algoritmo empregado na busca e seleção dos estudos, nesta pesquisa, é apresentado na figura 1, a seguir.

\section{Busca nas Bases de Dados \\ PubMed e Periódicos da Capes}

\section{Combinação entre os DeCS e \\ palavras-chave \\ (período de 2005 a 2020):}

Microneedling, skin, roller, rejuvenation, drug delivery

Manuscritos encontrados $\mathbf{N}=\mathbf{2 2 6}$

\section{$\downarrow$}

\begin{tabular}{|c|}
\hline \multicolumn{1}{|c|}{$\begin{array}{c}\text { Seleção Inicial de publicações com } \\
\text { temática relacionada ao estudo } \\
\text { (Screening inicial) } \\
\text { (N=210) }\end{array}$} \\
\hline \multicolumn{1}{|c|}{ Exclusões Diversas } \\
(N=203) \\
$\checkmark$ Duplicadas ( $\mathrm{n}=8$ ) \\
$\checkmark$ Estudos alheios aos objetivos da pesquisa ( $\mathrm{n}=139)$ \\
$\checkmark$ Estudos com textos incompletos ( $\mathrm{n}=56)$ \\
\hline \multicolumn{2}{|c|}{$\downarrow$} \\
\hline Artigos incluídos nesta revisão (N=23)
\end{tabular}

Figura 1. Algoritmo adotado para a busca e seleção de artigos na elaboração desta revisão.

\section{Resultados e Discussão}

A partir das análises realizadas, seguindo os critérios de inclusão acima descritos, foram encontrados 226 artigos no total, incluindo as bases de dados Portal de Periódicos Capes e PubMed, sendo que 203 trabalhos foram excluídos por não estarem relacionados aos objetivos do presente estudo, restando 23 referências que foram analisadas na íntegra.

Os trabalhos incluídos no estudo tratam da técnica do microagulhamento, da sua associação com o drug delivery, os mecanismos de ação, efeitos biológicos e eficácia do procedimento estético no rejuvenescimento cutâneo.

\section{Mecanismos de ação e efeitos biológicos}

O microagulhamento é realizado sobre a pele usando uma 
variedade de dispositivos, que podem ser divididos em: acoplados manual e motorizado. $O$ dispositivo manual é composto por um cilindro de polietileno contendo diversas microaguIhas estéreis em aço inoxidável. Os dispositivos motorizados consistem em uma peça de mão elétrica e uma unidade de cartucho de agulha descartável que se move, em várias direções sobre a pele ${ }^{7}$.

O microagulhamento cria mais de 500.000 canais em 5 minutos, até a derme, que é a maior obstrução à penetração de ingredientes ativos ${ }^{8}$. $O$ dispositivo pode ser passado sobre a área de tratamento de dez a quinze vezes, em quatro direções (vertical, horizontal e duas diagonais). Até que ocorra sangramento e exsudação pontual².

Os discos agulhados criam um dano mínimo à derme sem a remoção da epiderme saudável, o que acontece com a aplicação de outras terapias estéticas para rejuvenescimento cutâneo. Pelo fato de a epiderme ficar intacta, o período de cicatrização é rápido?.

O microagulhamento pode ocorrer de duas maneiras: estimulando a produção natural de colágeno (PNC) ou através da indução percutânea de colágeno (IPC), que ocorre através da resposta do processo inflamatório, que facilita o Sistema de Acesso Transdermal de Ingredientes (SATI), conhecido como "Drug Delivery", o qual aumenta da permeabilidade cutânea aos ativos cosméticos ${ }^{10}$.

As agulhas criam microcanais sobre a pele, que facilitam a permeação das formulações e cosméticos, quando aplicados em sequência penetram de forma mais rápida e eficaz. Os ativos presentes nos produtos alcançam partes mais profundas da pele, tendo uma maior eficiência do que quando aplicado somente de maneira tópica ${ }^{10}$.

A injúria provocada pelo microagulhamento, desencadeia a perda da integridade do tecido, formando uma nova produção de fibras colágenas afim de reparar as fibras danificadas, a dissociação dos queratinócitos, a liberação de citocinas por células da derme, gerando uma vasodilatação local com melhora da perfusão sanguínea tecidual, induzindo a melhora da oxigenação e nutrição tecidual ${ }^{12}$.

As microlesões na derme papilar estimulam a vasodilatação e induzem um sangramento superficial que atua como estímulo para desencadear o processo inflamatório e de cicatrização, estimulando a libração de diversos fatores de crescimento (fatores de crescimento derivados de plaquetas, fator de crescimento transformador alfa-TGFa, fator de crescimento transformador beta-TGF $\beta$, Interleucina-1, fator de necrose tumoral-TNF, etc.) que por sua vez estimulam a proliferação de fibroblastos e a síntese de colágeno III e I. Com a conversão de colágeno tipo III em tipo I, há uma contração na rede de colágeno, o que reduz a frouxidão da pele e suaviza cicatrizes cutâneas e rítides ${ }^{13-15}$.

Este processo de microlesões resulta em neocolagênese que preenche os locais da cicatriz cutânea, bem como induz a repigmentação através da melhora do suprimento sanguíneo. O microagulhamento resulta também em neoangiogênese, ou seja, formação de novos vasos sanguíneos no tecido tratado. O remodelamento tissular persiste por meses após o procedimento ${ }^{13-15}$. Os principais mecanismos de ação e efeitos biológicos do microagulhamento em tratamentos de rejuvenescimento cutâneo estão resumidos e apresentados do quadro 1 .

Quadro 1. Principais mecanismos de ação e efeitos biológicos do microagulhamento em tratamentos de rejuvenescimento cutâneo.

\begin{tabular}{|c|c|}
\hline Mecanismos de ação & Efeitos biológicos \\
\hline $\begin{array}{l}\checkmark \text { Criação de } \\
\text { microlesões na pele. } \\
\checkmark \text { Lesões atingem } \\
\text { diferentes camadas da } \\
\text { pele. } \\
\checkmark \text { Estímulo mecânico de } \\
\text { células cutâneas com } \\
\text { as agulhas } \\
\checkmark \text { Lesão ou ruptura de } \\
\text { capilares sanguineos } \\
\checkmark \text { Injúria tecidual de } \\
\text { queratinócitos e } \\
\text { fibroblastos. } \\
\checkmark \text { Ativação de } \\
\text { queratinócitos e } \\
\text { fibroblastos da pele. }\end{array}$ & $\begin{array}{l}\checkmark \text { Facilita a permeação } \\
\text { de cosméticos e } \\
\text { formulações na pele. } \\
\checkmark \text { Ativação de células } \\
\text { imunológicas da pele. } \\
\checkmark \text { Estimula a proliferação } \\
\text { de queratinócitos e a } \\
\text { angiogênese. } \\
\checkmark \text { Indução de um } \\
\text { processo inflamatório } \\
\text { controlado. } \\
\checkmark \text { Indução da produção } \\
\text { de colágeno e } \\
\text { elastina. } \\
\checkmark \text { Remodelação cutânea }\end{array}$ \\
\hline
\end{tabular}

Fonte: Lima et al (2013) ${ }^{6}$; Albano et al. $(2018)^{12}$; Liebl e Kloth $(2012)^{15}$.

As citocinas Fator de Crescimento Transformador (do inglês: Transforming growth fator - TGF) TGF- $\beta 1$ e o $\beta 2$, são liberadas por leucócitos cutâneos (Ex.: Macrófagos), após estímulo com as agulhas do microagulhamento na pele. Estas tendem a mostrar regulação positiva após duas semanas, mas apenas uma expressão fraca permanece após quatro semanas, enquanto o $\beta 3$ permanece otimizado nas oito semanas seguintes ao tratamento ${ }^{7}$.

A descoberta adicional de que o microagulhamento regula o TGF- $\beta 3$ sobre o TGF- $\beta 1$ e o TGF- $\beta 2$ (o último responsável 
pela cicatrização fibrótica) justifica ainda mais o seu uso no tratamento de rejuvenescimento ${ }^{16}$.

Curiosamente, modalidades de microagulhamento têm sido propostas como técnicas capazes de regular a pigmentação anormal, alterando o padrão de secreção do hormônio estimulador de melanócitos (MSH) e da Interleucina-10 (IL-10). A IL-10 é uma citocina anti-inflamatória liberada por leucócitos da pele, duas semanas após o procedimento e se torna indetectável após 4-8 semanas?

\section{O uso do microagulhamento no rejuvenescimento cutâneo}

Estudo realizado Aust e colaboradores (2008)8, na Alemanha, envolvendo indivíduos apresentando rugas, cicatrizes e estrias, utilizou de uma a quatro sessões de microagulhamento com aplicação tópica de vitamina A e C para o rejuvenescimento facial dos participantes. A avaliação dos pacientes, após o procedimento de microagulhamento, revelou uma melhoria de $60 \%$ a $80 \%$ na aparência da pele, exames histológicos, em 20 pacientes, demonstraram um aumento significativo de colágeno e elastina seis meses após o tratamento, e um aumento de $40 \%$ na espessura da epiderme um ano após o tratamento. Estes resultados evidenciam os benefícios do microagulhamento com drug delivery para indução percutânea de colágeno e rejuvenescimento cutâneo. A utilização adequada do tamanho das agulhas do equipamento de microagulhamento de acordo com a patologia estética a ser tratada é apresentada no quadro 2.

Quadro 2. Utilização do tamanho adequado de agulhas do equipamento de microagulhamento de acordo com o tratamento da patologia estética.

\begin{tabular}{|c|c|}
\hline $\begin{array}{c}\text { Patologia/Disfunção } \\
\text { estética cutânea }\end{array}$ & $\begin{array}{c}\text { Tamanho das agulhas } \\
(\mathrm{mm})\end{array}$ \\
\hline $\begin{array}{l}\text { Redução no crescimento } \\
\text { de cabelos }\end{array}$ & 0,3 \\
\hline Rugas finas & 1 a 1,5 \\
\hline $\begin{array}{l}\text { Rugas médias / } \\
\text { profundas }\end{array}$ & 1,5 a 2,5 \\
\hline Fotoenvelhecimento & 1,0 a 1,5 \\
\hline Flacidez tissular & 1,5 e áreas sensíveis 0,5 \\
\hline Manchas & 0,5 a 1,0 \\
\hline $\begin{array}{l}\text { Cicatrizes de acne / } \\
\text { queimaduras }\end{array}$ & 1,5 a 2,5 \\
\hline $\begin{array}{l}\text { Cicatrizes e estrias } \\
\text { cutâneas }\end{array}$ & 1,0 a 1,5 \\
\hline
\end{tabular}

Fonte: Negrão (2017) ${ }^{10}$; Bastos et al. (2019) ${ }^{11}$.
Foi realizado em estudo de acordo com Alessa e Bloom $(2020)^{3}$ avaliações clínicas e histológicas em 10 pacientes submetidos a 6 tratamentos com microagulhamento usando um Dermaroller e os resultados mostraram melhora significativa nas rugas, textura da pele e satisfação geral, bem como aumento de colágeno e tropoelastina nas amostras de biópsia. Melhoria estatisticamente significativa nos sinais de foto envelhecimento (rugas, flacidez e textura) também foi observada em outro estudo usando um dispositivo de microagulhamento motorizado.

Um estudo realizado na Índia em 2009, por Imran Majid ${ }^{14}$, utilizou o microagulhamento para o tratamento de 36 indivíduos com cicatrizes atróficas faciais. Observou-se, ao final do tratamento, uma excelente resposta ao microagulhamento por 26 pacientes $(72,2 \%)$, seis pacientes apresentaram boa resposta $(16,7 \%)$ e quatro $(11,1 \%)$ não demonstrando resposta significativa ao tratamento.

Estudos clínicos e histológicos relatados por Alessa e Bloom (2020) ${ }^{3}$ demonstraram a eficácia do microagulhamento para a remodelação tecidual; neocolagênese por meio da contração da fibrila de colágeno e melhora clínica foi observada na área periorbital (diminuição das rugas periorbitais, melhora da posição da sobrancelha), face média / face inferior (dobras nasolabiais, linhas de marionete, papada, flacidez sob o queixo) e flacidez do pescoço.

El-Domyati e colaboradores (2015) ${ }^{17}$ analisaram um estudo, sobre a avaliação dos resultados clínicos dos pacientes que mostrou uma melhora notável na resposta ao tratamento com microagulhamento na terapia para o rejuvenescimento cutâneo. As melhorias na aparência das rugas e na textura da pele aumentaram de 20-25\% (melhora leve) em um mês de tratamento (após duas sessões) para boa melhora (55-65\% e 55-60\%; respectivamente) em três meses após o tratamento. Enquanto isso, a satisfação do paciente foi relatada como moderada (35-45\%) em um mês de tratamento e, em seguida, aumentou para $80-90 \%$ (muito boa) em três meses pós tratamento. Após o microagulhamento da pele, os pacientes apresentaram eritema e edema transitórios, que desapareceram dois dias após o tratamento.

Fernandes e Signorini (2008) ${ }^{13}$ demonstraram que a técnica de microagulhamento é eficiente no tratamento de sinais de envelhecimento, assim como nos tratamentos de cicatrizes de acne e de queimaduras. Reforçando a ideia de que a indução percutânea de colágeno é efetiva no tratamento do envelhecimento.

Fabbrocini e colaboradores $(2011)^{9}$ realizaram um estudo utilizando o microagulhamento a fim de rejuvenescer a pele 
do pescoço de oito indivíduos. Com duas sessões de microagulhamento, observaram uma melhora de $90 \%$ dos pacientes, comprovando que o tratamento gera resultados positivos.

De acordo com El-Domyati e colaboradores $(2015)^{17}$ foi demonstrado em uma análise retrospectiva de 480 pacientes que a terapia de indução percutânea de colágeno é um método seguro e bem-sucedido para o rejuvenescimento da pele, e nenhum dos pacientes desenvolveu distúrbio pigmentar pós-operatório.

\section{O microagulhamento associado ao Drug delivery}

De acordo com Albano e colaboradores $(2018)^{12}$ ao realizar as micropunturas no tecido dérmico, as agulhas abrem canais que ligam a derme ao meio externo, podendo aumentar a permeação dos ativos em até 80 vezes. Estes canais permitem que a introdução e absorção de ativos de uso tópico, seja mais fácil e rapidamente depositada na camada mais profunda da pele. $\mathrm{O}$ uso do ativo pode ser feito antes, durante ou após o microagulhamento.

Em geral, moléculas maiores que 500 kDA não podem entrar no estrato córneo, a menos que seja criada uma via de entrada, como por exemplo, por meio do microagulhamento. O microagulhamento perfura a pele em uma profundidade controlada, o que causa cicatrização de feridas promovendo formação de colágeno e neovascularização. Ocorre migração e proliferação de fibroblastos, resultando na formação da matriz intercelular, e deposição de colágeno tipo $1{ }^{11}{ }^{18}$.

Em um relato de Alessa e Bloom (2020) ${ }^{3}$ foi realizado um ensaio clínico randomizado com 100 sujeitos do sexo masculino com alopecia androgenética moderada a grave, os sujeitos randomizados para o grupo de tratamento que receberam o microagulhamento combinado com a aplicação tópica de minoxidil tiveram resultados superiores estatisticamente significativos no crescimento do cabelo em comparação com indivíduos que receberam somente minoxidil.

Segundo Aust e colaboradores $(2008)^{8}$, o uso das vitaminas $\mathrm{A}$ e $\mathrm{C}$ associadas ao microagulhamento favorece $\mathrm{O}$ desenvolvimento de fibras de colágeno na pele. A vitamina A é um ácido retinoico capaz de controlar a liberação de citocinas como o TGF- $\beta 3$, TGF- $\beta 1$ e TGF- $\beta 2$ levando ao aumento da produção colágeno com padrão de retículo regenerativo, em vez da deposição paralela de colágeno cicatricial encontrado com a cicatrização. A vitamina $C$ é essencial para a produção de colágeno normal. $A$ vitamina $A$ associada à vitamina $C$ estimulam os fibroblastos a produzir colágeno e melhorar o rejuvenescimento da pele.

Alessa e Bloom (2020)ํaa analisaram ensaios clínicos controlados em tratamento estético facial para rejuvenescimento onde a face foi dividida comparando microagulhamento sozinho, com microagulhamento combinado com plasma rico em plaquetas (PRP) para cicatrizes de acne, mostraram resultados superiores no último grupo combinado (microagulhamento PRP). Infere-se que resultados foram produzidos pelos efeitos combinados de fatores de crescimento desencadeados pelas microlesões cutâneas de microagulhamento, bem como fatores de crescimento contidos no PRP. Os principais ativos associados ao drug delivery nos procedimentos de microagulhamento estão resumidos e apresentados no quadro 3.

Quadro 3. Principais ativos associados ao drug delivery nos procedimentos estéticos de microagulhamento.

\begin{tabular}{|c|c|}
\hline ATIVOS & FUNÇÃO \\
\hline Vitamina A (Retinol) & $\begin{array}{l}\text { Protege a pele contra a degradação do } \\
\text { colágeno e aumenta sua síntese; promove } \\
\text { renovação celular da pele; diminui } \\
\text { imperfeições e manchas; possui função de } \\
\text { regulação no crescimento e na atividade } \\
\text { das células epiteliais; favorece a síntese de } \\
\text { glicosaminoglicanas (GAG) e, portanto, a } \\
\text { hidratação da pele. }\end{array}$ \\
\hline $\begin{array}{c}\text { Vitamina C (Ácido } \\
\text { ascórbico) }\end{array}$ & $\begin{array}{l}\text { Estimula a formação de colágeno através } \\
\text { da biossíntese de fibras colágenas; é um } \\
\text { cofator fundamental na hidroxilação da } \\
\text { prolina e lisina, aminoácidos essenciais para } \\
\text { estrutura, manutenção e função do colágeno; } \\
\text { reduz a síntese de melanina; é um poderoso } \\
\text { antioxidante que aumenta os níveis de RNA } \\
\text { mensageiro pró-colágeno tipo I e III. }\end{array}$ \\
\hline $\begin{array}{l}\text { Vitamina E } \\
\text { (Tocoferol) }\end{array}$ & $\begin{array}{l}\text { É um antioxidante lipossolúvel; reduz a ação } \\
\text { da enzima metaloproteinase responsável pela } \\
\text { degradação das fibras colágenas. }\end{array}$ \\
\hline $\begin{array}{l}\text { Nicotinamida } \\
\text { (Vitamina B3) }\end{array}$ & $\begin{array}{l}\text { É um ativo multifuncional que aumenta a } \\
\text { hidratação da pele e a síntese de queratina; } \\
\text { estimula proteínas filagrina e involucrina, } \\
\text { que são de extrema importância para a } \\
\text { barreira epidérmica; estimula a síntese } \\
\text { de colágeno; reduz lesões causadas pela } \\
\text { acne melhorando a aparência da pele e } \\
\text { regula a produção sebácea; possui efeito } \\
\text { despigmentante decorrente da capacidade } \\
\text { de inibir a transferência de melanossomas dos } \\
\text { melanócitos para os queratinócitos. }\end{array}$ \\
\hline Vitamina B5 & $\begin{array}{l}\text { Age como hidratante, melhorando a } \\
\text { hidratação do estrato córneo, reduzindo a } \\
\text { perda de água transepidérmica mantendo a } \\
\text { pele macia e elástica. }\end{array}$ \\
\hline
\end{tabular}




\begin{tabular}{|c|l|}
\hline Manganês & $\begin{array}{l}\text { É essencial para a assimilação do mecanismo } \\
\text { dos glucídios e lipídeos. Atua na proteção } \\
\text { da barreira lipídica e controla a elaboração } \\
\text { de várias enzimas para o metabolismo da } \\
\text { vitamina B1 e B3 e vitamina C. }\end{array}$ \\
\hline Silício & $\begin{array}{l}\text { Atua na manutenção e na conservação da } \\
\text { estrutura da derme; promove uma recuperação } \\
\text { dos tecidos danificados. }\end{array}$ \\
\hline Zinco & $\begin{array}{l}\text { É fundamental para a oxigenação celular e } \\
\text { reconstituição da membrana celular; protege } \\
\text { os ácidos nucleicos (RNA-DNA) das células e ao } \\
\text { mesmo tempo garante integridade molecular. }\end{array}$ \\
\hline Ácido Hialurônico & $\begin{array}{l}\text { É uma glicosaminoglicana que mantem a } \\
\text { pele em equilíbrio; alta capacidade de atrair e } \\
\text { manter grandes quantidades de água na pele, } \\
\text { aumenta a hidratação superficial e profunda; } \\
\text { cicatrizante; resedificante; antioxidante. }\end{array}$ \\
\hline $\begin{array}{l}\text { Fatores de } \\
\text { Crescimento }\end{array}$ & $\begin{array}{l}\text { São produzidos naturalmente pelas células } \\
\text { responsáveis pela comunicação celular; }\end{array}$ \\
\hline
\end{tabular}

Fonte: Adaptado de Negrão $(2017)^{10}$.

Um ensaio controlado investigando o tratamento do melasma foi conduzido entre 20 indivíduos que receberam tratamento facial; um lado tinha microagulhas combinado com um soro despigmentante e o outro lado tinha o soro sozinho. Os resultados deste estudo mostraram uma redução estatisticamente significativa na área de melasma e pontuação do índice de gravidade no lado do tratamento combinado em comparação com o lado tratado apenas com o soro ${ }^{3}$.

Segundo Bernardi e Ognibeni (2019) ${ }^{19}$ o uso do microagulhamento isolado e do microagulhamento com fator de crescimento insulínico, com intervalo de 30 dias entre as aplicações, resultou na redução de rugas finas nas regiões periorbital, frontal e sulco nasogeniano nos participantes do estudo. Os microcanais provocados pelo dermaroller facilitam a absorção dos ativos, aumentando assim a penetração de moléculas maiores em até $80 \%$, potencializando os resultados do procedimento.

Estudo controlado e assistido no tratamento de rejuvenescimento da face anterior da pele do tórax, foram incluídas neste estudo 22 mulheres com a faixa etária de 55 anos fototipo de Fitzpatrick de I a IV16 e escala de envelhecimento de Glogau 2 a 4 . Foi feito um acompanhamento de três meses com 30 dias de intervalo. A avaliação da eficácia clínica demonstrou melhora significativa em $100 \%$ das pacientes tratadas. A análise estatística mostrou melhora de $28 \%$ no rejuvenescimento global da pele da região anterior do tórax ${ }^{20}$.

\section{Cuidados na aplicação do microagulhamento}

Segundo Negrão (2017) ${ }^{10}$ a aplicação do microagulhamento poderá ser realizada com ou sem anestésico. É imprescindível higienizar a área com clorexidina alcoólica. Verificar o tamanho da agulha perante o distúrbio estético a ser tratado. Ele ainda afirma que a periodicidade também será proporcional ao tamanho das agulhas utilizadas para o procedimento, ou seja, quanto maior a agulha, maior será o espaço entre as aplicações. Em média, o intervalo seria de 30 dias, porém pode-se dar um espaço de 45 a 60 dias.

A profundidade de penetração das agulhas é determinada pelo profissional, dependendo da área específica e da espessura da pele a ser tratada, com agulhas mais profundas para pele grossa e menor profundidade para pele fina ou delicada, como a área periorbital ${ }^{3}$.

A utilização da forma correta do roller é com o posicionamento entre os dedos indicador e polegar, para melhor controle da força exercida com o polegar, com o uso de uma pressão moderada para uma melhor eficácia. Observou-se que o emprego de uma força maior que a necessária pode levar a danos em estruturas anatômicas mais profundas, com um resultado menor que o esperado, bem como causar nos pacientes uma dor incômoda no tratamento ${ }^{5,6}$. O trauma feito pela agulha deve atingir a profundidade na pele de 1 a $3 \mathrm{~mm}$, preservando a epiderme, causando edema na área tratada e hemostasia praticamente imediata ${ }^{5,6}$.

Na primeira semana do tratamento com o microagulhamento, recomenda-se cobrir a área tratada com uma camada muito fina de uma pomada oclusiva que contenha vitaminas A e D. Não expor as áreas tratadas ao sol por dez dias e, posteriormente, usar um protetor solar com um elevado fator de proteção. Esperar dois ou três dias para a aplicação de maquiagem ${ }^{5,6,8,21}$.

A técnica de microagulhamento proporciona resultados satisfatórios em disfunções estéticas auxiliando no rejuvenescimento cutâneo, melhora nas cicatrizes de acne, clareamento de manchas, melhorando a circulação da área tratada bem como o aspecto geral do tecido. O número de sessões varia de acordo com a disfunção tratada e o caso clínico de cada paciente ${ }^{21}$.

A vantagem do microagulhamento é que é feito em consultório, tem custo baixo, é bem tolerado, com período curto de recuperação e com pouca dor. A expectativa é de melhora após primeira sessão dependendo do tamanho da agulha ${ }^{8}$.

\section{Efeitos adversos e complicações}

Para McCrudden e colaboradores (2015) ${ }^{22}$ os efeitos potenciais de longo prazo da aplicação de microagulhamento ou 
mesmo da aplicação repetida de microagulhamento na pele são atualmente desconhecidos. Como acontece com qualquer nova tecnologia, o uso inadequado pode causar problemas. Com referência específica aos dispositivos de microagulhamento, foram relatados três casos de hipersensibilidade alérgica granulomatosa e sistêmica em pacientes do sexo feminino, após a aplicação de um produto tópico não estéril, em todos os três casos. Os efeitos colaterais deletérios testemunhados foram determinados como sendo devidos à tatuagem intradérmica inadequada da pele com produtos tópicos antigênicos. O uso combinado desses produtos tópicos com aplicação microagulhamento não é licenciado, e, portanto, a entrega artificialmente aprimorada desses produtos para a derme, neste relato, resultou nas reações de hipersensibilidade observadas. Em todos os casos, a recuperação total ou parcial foi alcançada após tratamentos com corticosteroides ou tetraciclina ${ }^{22}$.

Segundo Lisa (2017) ${ }^{23}$ estudos in vitro demonstraram que há uma passagem transdérmica de microrganismos através dos microporos após o microagulhamento, mas não há risco aumentado de infecção quando o microagulhamento é realizado com medidas higiênicas padrão, como um dispositivo estéril e desinfecção da pele. Até mostrou menos contaminação em comparação com agulhas intradérmicas.

De acordo com Negrão (2017) ${ }^{10}$, as complicações devido ao microagulhamento podem acontecer por fatores diversos: escolha do equipamento é importante sempre observar se o dispositivo tem liberação da Agência Nacional de Vigilância Sanitária (Anvisa), execução inadequada, uso de substância com potencial alergênico, má associação terapêutica, entre outros. Algumas reações, no entanto, são inerentes à técnica como: sangramento durante a sessão, hiperemia, dor local, descamação e edema. São características de qualquer processo inflamatório. Poderá haver também marcas de arranhões quando o equipamento for arrastado ou se o paciente se movimentar muito durante o procedimento. A hipercromia pós inflamatória ocorrerá caso haja exposição solar. A pele também poderá liberar exsudato seroso logo após a aplicação e depois cessa.

De acordo com Lisa (2017) ${ }^{23}$ esses eventos ilustram que, embora a microagulhamento seja uma opção terapêutica simples e minimamente invasiva, são necessárias diretrizes claras e baseadas em evidências para evitar esses efeitos colaterais ou complicações indesejadas.

\section{Conclusão}

O microagulhamento é um tratamento estético seguro, minimamente invasivo e eficaz para variadas condições dermatológicas, incluindo acne e outras cicatrizes, rítides e estrias cutâneas. Dada a sua rápida recuperação pós-tratamento conveniente, perfil de efeitos colaterais limitado e resultados clínicos significativos, o microagulhamento é uma valiosa alternativa a procedimentos estéticos mais invasivos.

Por meio das micro lesões cutâneas, causadas pelo deslizamento das agulhas, ocorre um grande aumento da permeação de ativos potencializadores e uma significante produção de colágeno e elastina sem causar grandes danos a pele, apresentando uma cicatrização rápida se comparada a outros tratamentos estéticos.

O uso de tecnologias de rolos com microagulhas para facilitar a entrega eficiente de produtos farmacêuticos na derme, resulta da ruptura de células do estrato córneo, reduzindo as funções desta barreira principal da pele, causando um aumento na absorção de compostos ativos farmacológicos, transdermicamente, estimulando, desta forma, uma maior produção de colágeno e elastina.

\section{Referências}

1. BergmannCLMS,LunardelliA.Usodemicroagulhamento e ácido retinóico para o tratamento de melasma. Revista Brasileira de Estética. 2018;6(3):78-88.

2. El-Domyati M, Abdel-Wahab H, Hossam A. Combining microneedling with other minimally invasive procedures for facial rejuvenation: a split-face comparative study. Int j dermatol. 2018;57(11):1324-1334.

3. Alessa D, Bloom J D. Microneedling Options for Skin Rejuvenation, Including Non-temperature controlled Fractional Microneedle Radiofrequency Treatments. Facial Plast Surg Clin. 2020;28(1):1-7.

4. Gold MH, Biron J, Thompson B. Randomized, singleblinded, crossover study of a novel wound dressing vs current clinical practice after percutaneous collagen induction therapy. J Cosmet Dermatol. 2019;18(2):524529.

Gupta J, Gill HS, Andrews SN, Prausnitz MR. Kinetics of skin resealing after insertion of microneedles in human subjects. J Control Release. 2011;154(2):148-155. DOl: 10.1016 / j. jconrel.2011.05.021.

5. Lima EVA, Lima MA, Takano D. Microneedling experimental study and classification of the resulting injury. Surg Cosmet Dermatol. 2013;5(2):110-4.

6. Iosifidis C, Goutos I. Percutaneous collagen induction (microneedling) for the management of non-atrophic scars: literature review. Scars, Burns \& Healing, 2019;5:1-11.

7. Aust MC. et al. Percutaneous collagen induction: minimally invasive skin rejuvenation without risk of hyperpigmentation-fact or fiction? Plastic and Reconstructive Surgery. 2008;122(5):1553-1563.

8. Fabbrocini $\mathrm{G}$, et al. Skin needling in the treatment of the aging neck. SKINmed. 2011;9(6):347-351. 
9. Negrão MMC. Microagulhamento bases fisiológicas e práticas. CR8 Editora, 2a edição; São Paulo, 2017.

10. Bastos LC, Nascimento TL, Pereira L. Aplicabilidade do microagulhamento associado a vitamina $C$ para rejuvenescimento facial. Revista Saúde em Foco. 2019;11:1045-1069.

11. Albano RPS, Pereira LP, Assis IB. Microagulhamento - a terapia que induz a produção de colágeno - revisão de literatura, Revista Saúde em Foco. 2018;10:455473.

12. Fernandes D, Signorini M. Combating photoaging with percutaneous collagen induction. Clin Dermatol. 2008;26(2):192-199.

13. Majid I. Microneedle therapy in atrophic facial scars: an objective assessment. Journal of Cutaneous and Aesthetic Surgery. 2009;2(1):26-30.

Liebl H, Kloth LC. Skin Cell Proliferation stimulated by microneedles. J Am Coll Wound Spec. 2012;4(1):2-6.

14. Auster TS, Yili MK. Microneedling of Scars: A Large Prospective Study with Long-Term Follow-U. Plast Reconstr Surg. Milan, Italy. 2019;145(5):1341-1349.

El-Domyati et al. Microneedling Therapy for Atrophic Acne Scars - An Objective Evolution. J Clin Aesthet Dermatol. 2015;8(7):36-42.

15. Aldag C, Teixeira DN, Leventhal PS. Skin rejuvenation using cosmetic products containing growth factors, cytokines and matrikines: A review of the literature. Clin Cosmet Investig Dermatol. 2016;9:411-419.

16. Bernardi MN, Ognibeni LCR. Uso do microagulhamento associado a princípios ativos do tratamento de cicatrizes de acne. Revista Uningá, Maringá. 2019;56(4):93-103.

17. Kalil PV, et al. Estudo comparativo, randomizado e duplo-cego do microagulhamento associado ao drug delivery para rejuvenescimento da pele da região anterior do tórax. Surgical \& Cosmetic Dermatology. 2015;7(3):211-216, 2015.

18. Piatti IL. Microagulhamento e fatores de crescimento. Revista Personalité, São Paulo.2013;16(8):22-25.

19. Mccrudden TCM, et al. Microneedle applications to improve skin appearance. Exp Dermatology. 2015;24(8):561-566.

20. Lisa R, et. al. Microneedling: Where are we now? A systematic review of the literature. British Journal of Plastic Surgery. 2017;71(1):1-14,2017. 